\title{
The spectrum of charmonium-like vector mesons in lattice QCD
}

TWQCD Collaboration: Ting-Wai Chiü ${ }^{* a}$,Tung-Han Hsieh ${ }^{b}$

${ }^{a}$ Department of Physics and Center for Theoretical Sciences, National Taiwan University, Taipei 10617, Taiwan

E-mail: twchiu@phys.ntu.edu.tw

${ }^{b}$ Research Center for Applied Sciences, Academia Sinica, Taipei 115, Taiwan

E-mail: thhsieh@twcp1.phys.ntu.edu.tw

We present the first lattice results of the spectrum of exotic vector mesons extracted from the molecular and diquark-antidiquark operators, with quark fields $(\mathbf{c q} \overline{\mathbf{c}} \overline{\mathbf{q}})$, and $(\mathbf{c s} \overline{\mathbf{c}} \overline{\mathbf{q}}) /(\mathbf{c q} \overline{\mathbf{c}} \overline{\mathbf{s}})$ respectively, in lattice QCD with exact chiral symmetry. Our results suggest that $X(3872)$ and $Y(4260)$ are in the spectrum of QCD, with $J^{P C}=1^{++}$and $1^{--}$respectively. Moreover, we obtain the spectrum of heavier exotic mesons with $(\operatorname{cs} \overline{\mathbf{c}} \overline{\mathbf{u}}) /(\mathbf{c u} \overline{\mathbf{c}} \overline{\mathbf{s}}),(\mathbf{c s} \overline{\mathbf{c}} \overline{\mathbf{d}}) /(\mathbf{c d} \overline{\mathbf{c}} \overline{\mathbf{s}}),(\mathbf{c s} \overline{\mathbf{c}} \overline{\mathbf{s}})$, and $(\mathbf{c} \overline{\mathbf{c}} \overline{\mathbf{c}})$, as the first theoretical predictions from lattice QCD.

XXIVth International Symposium on Lattice Field Theory

July 23-28, 2006

Tucson, Arizona, USA

${ }^{*}$ Speaker. 


\section{Introduction}

Since the discovery of $D_{s}(2317)$ [1] by BABAR in April 2003, a series of new heavy mesons with open-charm and closed-charm have been observed by Belle, CDF, CLEO, BABAR, and BES. Among these new heavy mesons, the most intriguing ones are the charmonium-like states, $X(3872)$ [2], $Y(3940)$ [3], $Y(4260)$ [4], $Z(3930)$ [5], and $X(3940)$ [6]. Evidently, one can hardly interpret all of them as orbital and/or radial excitations in the charmonium spectrum. Thus it is likely that some of them are exotic (non- $q \bar{q}$ ) mesons (e.g., molecule, diquark-antidiquark, and hybrid meson). Theoretically, the central question is whether the spectrum of QCD possesses these resonances, with the correct quantum numbers, masses, and decay widths. Now the most viable approach to tackle this problem from the first principles of QCD is the lattice QCD with exact chiral symmetry [7, 8, 9, 10].

Recently, we have investigated the mass spectrum of closed-charm exotic mesons with $J^{P C}=$ $1^{--}[11]$ and $1^{++}[12]$ respectively, in the framework of lattice QCD with exact chiral symmetry. By constructing molecular and diquark-antidiquark operators with quark content $(\mathbf{c q} \overline{\mathbf{c}} \overline{\mathbf{q}})$, we measure the time-correlation function of each operator, and fit to the usual formula

$$
W\left\{e^{-m a t}+e^{-m a(T-t)}\right\}
$$

to extract the mass $(m)$ and the spectral weight $(W)$ of the lowest-lying state overlapping with the exotic meson operator. From the ratio of the spectral weights of two spatial volumes $\left(20^{3}\right.$ and $\left.24^{3}\right)$ with the same lattice spacing, we can infer whether this state is a resonance $\left(W_{20} / W_{24} \simeq 1\right)$ or 2-particle scattering state $\left(W_{20} / W_{24} \simeq(24 / 20)^{3}=1.728\right)$.

Our results suggest that $Y(4260)$ and $X(3872)$ are in the spectrum of QCD, with $J^{P C}=1^{--}$ and $1^{++}$respectively, and both with quark content $(\mathbf{c u c} \overline{\mathbf{c}})$. Note that we have been working in the isospin limit $\left(m_{u}=m_{d}\right)$, thus our results [11,12] cannot exclude the possibility of the existence of exotic mesons with quark content $(\mathbf{c d} \overline{\mathbf{c}} \overline{\mathbf{d}})$, even though we cannot determine their mass differences from those with (cuc̄ū). Moreover, we also observe heavier exotic mesons with quark contents $(\mathbf{c s} \overline{\mathbf{s}})$ and $(\mathbf{c} \overline{\mathbf{c}} \overline{\mathbf{c}})$, for $J^{P C}=1^{++}[12]$, and $1^{--}$[11] respectively.

Now if the spectrum of QCD does possess exotic mesons with quark content ( $\mathbf{c q} \overline{\mathbf{c}} \overline{\mathbf{q}})$, then it is likely that there are also exotic mesons with other quark contents, e.g., $(\mathbf{c q} \overline{\mathbf{c}} \overline{\mathbf{s}})$ and $(\mathbf{c s} \overline{\mathbf{c}} \overline{\mathbf{q}})$. However, in general, whether any combination of two quarks and two antiquarks can emerge as a hadronic state relies on the nonperturbative dynamics between these four quarks. Recently, we have investigated the lowest-lying mass spectrum of molecular and diquark-antidiquark operators with quark content $(\mathbf{c s} \overline{\mathbf{c}} \overline{\mathbf{q}}) /(\mathbf{c q} \overline{\mathbf{c}} \overline{\mathbf{s}})$ and $J^{P}=1^{+}[13]$. Our results suggest that there exists a $J^{P}=1^{+}$ resonance around $4010 \pm 50 \mathrm{MeV}$. It is interesting to see whether this state will be observed by high energy experiments.

\section{Lattice setup}

To implement exact chiral symmetry on the lattice, we consider the optimal domain-wall fermion proposed by Chiu [14]. From the generating functional for $n$-point Green's function of the quark fields, the valence quark propagator in background gauge field can be derived as [14]

$$
\langle q(x) \bar{q}(y)\rangle=\left(D_{c}+m_{q}\right)_{x, y}^{-1}, \quad D_{c}=2 m_{0} \frac{1+\gamma_{5} S\left(H_{w}\right)}{1-\gamma_{5} S\left(H_{w}\right)}
$$


where $m_{q}$ is the bare quark mass, $m_{0}$ is a parameter in the range $(0,2), S\left(H_{w}\right)$ is equal to the Zolotarev approximation of the sign function of $H_{w}\left(H_{w}=\gamma_{5} D_{w}\right.$, and $D_{w}$ is the standard Wilson Dirac operator minus $m_{0}$ ). In the limit $N_{s} \rightarrow \infty$ (where $N_{s}+2$ is the number of sites in the 5th dimension), $D_{c}$ is exactly chirally symmetric, i.e., $D_{c} \gamma_{5}+\gamma_{5} D_{c}=0$. In the continuum limit, the valence quark propagator $\left(D_{c}+m_{q}\right)^{-1} \rightarrow\left[\gamma_{\mu}\left(\partial_{\mu}+i g A_{\mu}\right)+m_{q}\right]^{-1}$. A salient feature of the valence quark propagator (2.1) is that the bare quark mass $m_{q}$ is well-defined for any gauge field configuration, unlike the Wilson quark propagator. In practice, we compute the valence quark propagator by the nested conjugate gradient

$$
\begin{aligned}
D\left(m_{q}\right) Z & =\left\{m_{q}+\left(m_{0}-m_{q} / 2\right)\left[1+\gamma_{5} S\left(H_{w}\right)\right]\right\} Z=\mathbf{I} \\
S\left(H_{w}\right) Z & =\sum_{l} b_{l} z_{l}, \quad\left(H_{w}^{2}+c_{l}\right) z_{l}=Z
\end{aligned}
$$

where $b_{l}$ and $c_{l}$ are Zolotarev coefficients [15, 16]. To attain the maximal efficiency of our system (CPU time and memory usage vs. the precision of the sign function $S\left(H_{w}\right)$ ), we use Neuberger's 2-pass algorithm [17, 18] in the inner conjugate gradient (2.3).

We generate 100 gauge configurations with single plaquette gauge action at $\beta=6.1$, for two lattice volumes $24^{3} \times 48$ and $20^{3} \times 40$, with the same lattice spacing. Fixing $m_{0}=1.3$, we project out 16 low-lying eigenmodes of $\left|H_{w}\right|$ and perform the nested conjugate gradient in the complement of the vector space spanned by these eigenmodes. For $N_{s}=128$, the weights $\left\{\omega_{s}\right\}$ are fixed with $\lambda_{\min }=0.18$ and $\lambda_{\max }=6.3$, where $\lambda_{\min } \leq \lambda\left(\left|H_{w}\right|\right) \leq \lambda_{\max }$ for all gauge configurations. For each configuration, point-to-point quark propagators are computed for 30 bare quark masses in the range $0.03 \leq m_{q} a \leq 0.8$, with stopping criteria $10^{-11}$ and $2 \times 10^{-12}$ for the outer and inner conjugate gradient loops respectively. Then the norm of the residual vector of each column of the quark propagator is less than $2 \times 10^{-11}$

$$
\left\|\left(D_{c}+m_{q}\right) Y-\mathbf{I}\right\|<2 \times 10^{-11}, \quad Y=\left\{1-m_{q} /\left(2 m_{0}\right)\right\}^{-1}\left\{Z-1 /\left(2 m_{0}\right)\right\},
$$

and the chiral symmetry breaking due to finite $N_{s}$ is less than $10^{-14}$,

$$
\sigma=\left|\frac{Z^{\dagger} S^{2} Z}{Z^{\dagger} Z}-1\right|<10^{-14},
$$

for every iteration of the nested conjugate gradient.

Then we measure the time-correlation functions of pseudoscalar and vector meson operators. We determine the inverse lattice spacing $a^{-1}=2.237(76) \mathrm{GeV}$ from the pion time-correlation function, with the experimental input of pion decay constant $f_{\pi}=131 \mathrm{MeV}$. The strange quark bare mass $m_{s} a=0.08$ and the charm quark bare mass $m_{c} a=0.80$ are fixed such that the corresponding masses extracted from the vector meson correlation function agree with $\phi(1020)$ and $J / \psi(3097)$ respectively [19, 20].

\section{Four-quark meson operators}

In QCD, there is no reason why any hadronic state composed of two quarks and two antiquarks cannot emerge as a resonance. Thus, it is interesting to see whether such four-quark mesons can exist in the spectrum of QCD, and also to identify them with any meson states observed in high 
energy experiments. To tackle this problem in the framework of lattice $\mathrm{QCD}$, one needs to construct interpolating operators which can have good overlap with the 4-quark meson states. Otherwise, their signals may not be unambiguously identified, due to the limitations in statistics, the lattice size, as well as the unphysically-heavy $\mathbf{u}(\mathbf{d})$ quark masses which have to be chirally extrapolated to their physical values. Further, for any lattice calculations in quenched approximation, one should avoid any operators (e.g., scalar operator $\overline{\mathbf{c}} \mathbf{q}$ ) which have strong quenched artifacts as $m_{q} \rightarrow m_{u, d}$.

In general, there are two ways to construct 4-quark meson operators: (i) molecular operator, and (ii) diquark-antidiquark operator. In the following, we only present the 4-quark operators which have good overlap (except $Y_{4}$ ) with the hadronic states in our investigations [11, 12, 13]. Explicitly, they are

$$
\begin{aligned}
J^{P C}=1^{--}: & \\
M_{3}(x) & =\frac{1}{\sqrt{2}}\left\{\left(\overline{\mathbf{q}} \gamma_{5} \gamma_{i} \mathbf{c}\right)_{x}\left(\overline{\mathbf{c}} \gamma_{5} \mathbf{q}\right)_{x}-\left(\overline{\mathbf{c}} \gamma_{5} \gamma_{i} \mathbf{q}\right)_{x}\left(\overline{\mathbf{q}} \gamma_{5} \mathbf{c}\right)_{x}\right\} \\
Y_{4}(x) & =\frac{1}{\sqrt{2}}\left\{\left(\mathbf{q}^{T} C \gamma_{5} \gamma_{i} \mathbf{c}\right)_{a x}\left(\overline{\mathbf{q}} C \gamma_{5} \overline{\mathbf{c}}^{T}\right)_{a x}+\left(\overline{\mathbf{q}} C \gamma_{5} \gamma_{i}^{T} \overline{\mathbf{c}}^{T}\right)_{a x}\left(\mathbf{q}^{T} C \gamma_{5} \mathbf{c}\right)_{a x}\right\} \\
J^{P C}=1^{++}: & M_{1}(x)=\frac{1}{\sqrt{2}}\left\{\left(\overline{\mathbf{q}} \gamma_{i} \mathbf{c}\right)_{x}\left(\overline{\mathbf{c}} \gamma_{5} \mathbf{q}\right)_{x}-\left(\overline{\mathbf{c}} \gamma_{i} \mathbf{q}\right)_{x}\left(\overline{\mathbf{q}} \gamma_{5} \mathbf{c}\right)_{x}\right\} \\
X_{4}(x) & =\frac{1}{\sqrt{2}}\left\{\left(\mathbf{q}^{T} C \gamma_{i} \mathbf{c}\right)_{a x}\left(\overline{\mathbf{q}} C \gamma_{5} \overline{\mathbf{c}}^{T}\right)_{a x}-\left(\overline{\mathbf{q}} C \gamma_{i}^{T} \overline{\mathbf{c}}^{T}\right)_{a x}\left(\mathbf{q}^{T} C \gamma_{5} \mathbf{c}\right)_{a x}\right\} \\
J^{P}=1^{+}: \quad & M_{s}(x)=\frac{1}{\sqrt{2}}\left\{\left(\overline{\mathbf{q}} \gamma_{i} \mathbf{c}\right)_{x}\left(\overline{\mathbf{c}} \gamma_{5} \mathbf{s}\right)_{x}-\left(\overline{\mathbf{c}} \gamma_{i} \mathbf{s}\right)_{x}\left(\overline{\mathbf{q}} \gamma_{5} \mathbf{c}\right)_{x}\right\} \\
D_{4}(x) & =\frac{1}{\sqrt{2}}\left\{\left(\mathbf{q} T C \gamma_{i} \mathbf{c}\right)_{a x}\left(\overline{\mathbf{s} C} \gamma_{5} \overline{\mathbf{c}}^{T}\right)_{a x}-\left(\overline{\mathbf{s} C} \gamma_{i}^{T} \overline{\mathbf{c}}^{T}\right)_{a x}\left(\mathbf{q}^{T} C \gamma_{5} \mathbf{c}\right)_{a x}\right\}
\end{aligned}
$$

where

$$
(\overline{\mathbf{q}} \Gamma \mathbf{Q})_{x}=\overline{\mathbf{q}}_{a \alpha x} \Gamma_{\alpha \beta} \mathbf{Q}_{a \beta x}
$$

denotes a meson operator with antiquark field $\overline{\mathbf{q}}$ coupling to quark field $\mathbf{Q}$ through the Dirac matrix $\Gamma$. Here $x,\{a, b, c\}$ and $\{\alpha, \beta\}$ denote the lattice site, color, and Dirac indices respectively. The diquark operator is denoted by

$$
\left(\mathbf{q}^{T} \Gamma \mathbf{Q}\right)_{a x}=\varepsilon_{a b c} \mathbf{q}_{b \alpha x} \Gamma_{\alpha \beta} \mathbf{Q}_{c \beta x}
$$

where $\varepsilon_{a b c}$ is the completely antisymmetric tensor, $C$ is the charge conjugation operator satisfying $C \gamma_{\mu} C^{-1}=-\gamma_{\mu}^{T}$ and $\left(C \gamma_{5}\right)^{T}=-C \gamma_{5}$. Thus the diquark transforms like color anti-triplet. For $\Gamma=$ $C \gamma_{5}$, it transforms like $J^{P}=0^{+}$, while for $\Gamma=C \gamma_{i}(i=1,2,3)$, it transforms like $1^{+}$.

\section{Results and discussions}

In Table 1, we summarize our results of the masses of the lowest-lying states of the 4-quark operators obtained in Refs. [11, 12, 13], where in each case, the first error is statistical, and the second one is our estimate of combined systematic uncertainty including: (i) possible plateaus (fit ranges) with $\chi^{2} /$ d.o.f. $<1$; (ii) the uncertainties in the strange quark mass and the charm quark 
mass; (iii) chiral extrapolation (for the entries containing $\mathrm{u} / \mathrm{d}$ quarks); and (iv) finite size effects (by comparing results of two lattice sizes). Note that we cannot estimate the discretization error since we have been working with one lattice spacing.

\section{1 $J^{P C}=1^{--}$states}

The molecular operator $M_{3} \sim\left\{\left(\overline{\mathbf{q}} \gamma_{5} \gamma_{i} \mathbf{c}\right)\left(\overline{\mathbf{c}} \gamma_{5} \mathbf{q}\right)-\left(\overline{\mathbf{c}} \gamma_{5} \gamma_{i} \mathbf{q}\right)\left(\overline{\mathbf{q}} \gamma_{5} \mathbf{c}\right)\right\}$ detects a resonance with mass 4238(31)(57) MeV in the limit $m_{q} \rightarrow m_{u, d}$, which is naturally identifed with $Y$ (4260). This suggests that $Y(4260)$ is indeed in the spectrum of QCD, with quark content (cuc̄ū) and $J^{P C}=1^{--}$.

For the diquark-antidiquark operator $Y_{4} \sim\left\{\left[\mathbf{q}^{T} C \gamma_{5} \gamma_{i} \mathbf{c}\right]\left[\overline{\mathbf{q}} C \gamma_{5} \overline{\mathbf{c}}^{T}\right]+\left[\mathbf{q}^{T} C \gamma_{5} \mathbf{c}\right]\left[\overline{\mathbf{q}} C \gamma_{5} \gamma_{i}^{T} \overline{\mathbf{c}}^{T}\right]\right\}$, it also detects a state with mass $4267(68)(83) \mathrm{MeV}$, in the limit $m_{q} \rightarrow m_{u}$. We suspect that this state might be the same resonance captured by the molecular operator $M_{3}$. However, we are not sure that this state is a resonance since the ratio of spectral weights $\left(R=W_{20} / W_{24}\right)$ for two different lattice volumes with the same lattice spacing deviates from one (the criterion for a resonance) with large errors as $m_{q} \rightarrow m_{u}$. It is plausible that such a deviation is due to the quenched artifacts which can be evaded if one incorporates internal quark loops.

Now, in the quenched approximation, our results suggest that $Y(4260)$ has a better overlap with the molecular operator $M_{3}$ than the diquark-antidiquark operator $Y_{4}$. Whether this implies that $Y(4260)$ behaves more likely as a $D_{1} \bar{D}$ molecule than a diquark-antidiquark meson is subjected to further investigations, especially those incorporating dynamical quarks.

For molecular and diquark-antidiquark operators with quark fields ( $\mathbf{c s} \overline{\mathbf{c}} \overline{\mathbf{s}})$, they both detect a resonance around $4450 \pm 100 \mathrm{MeV}$, and for the molecular operator with $(\mathbf{c c} \overline{\mathbf{c}} \overline{\mathbf{c}})$, it detects a resonance around $6400 \pm 50 \mathrm{MeV}$. These serve as predictions from lattice QCD.

\section{2 $J^{P C}=1^{++}$states}

Both the molecular operator $M_{1}$ and the diquark-antidiquark operator $X_{4}$ detect a $1^{++}$resonance around $3890 \pm 30 \mathrm{MeV}$ in the limit $m_{q} \rightarrow m_{u, d}$, which is naturally identified with $X(3872)$.

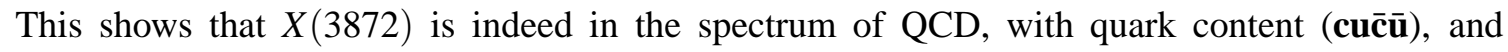
$J^{P C}=1^{++}$. It is interesting to see that $X(3872)$ has good overlap with the molecular operator $M_{1}$ as well as the diquark-antidiquark operator $X_{4}$. This is in contrast to the case of $Y(4260)$, in which $Y(4260)$ seems to have better overlap with the molecular operator $M_{3}$ than any diquarkantidiquark operators. This seems to suggest that $X(3872)$ is more tightly bounded than $Y(4260)$. It would be interesting to see whether this picture persists even for unquenched QCD.

For $m_{q}=m_{s}$, heavier exotic meson resonance with $J^{P C}=1^{++}$is also detected, with quark content $(\boldsymbol{c s} \overline{\mathbf{c}} \overline{\mathbf{s}})$ around $4100 \pm 50 \mathrm{MeV}$. This serves as a prediction from lattice QCD.

\section{3 $J^{P C}=1^{+}$states}

Both the molecular operator $M_{s}$ and the diquark-antidiquark operator $D_{4}$ detect a $1^{+}$resonance around $4010 \pm 50 \mathrm{MeV}$ in the limit $m_{q} \rightarrow m_{u, d}$. Since its mass is just slightly above Y(3940), high energy experiments should be able to see whether such a resonance, say, $X_{s}$, exists in some decay channels, e.g., $X_{S} \rightarrow K \pi J / \Psi$, in the near future. 


\begin{tabular}{|c|c|c|c|c|}
\hline$J^{P C}\left(J^{P}\right)$ & Operator & Mass $(\mathrm{MeV})$ & $\mathrm{R} / \mathrm{S}$ & Candidate \\
\hline $1^{--}$ & $\frac{1}{\sqrt{2}}\left[\left(\overline{\mathbf{u}} \gamma_{5} \gamma_{i} \mathbf{c}\right)\left(\overline{\mathbf{c}} \gamma_{5} \mathbf{u}\right)-\left(\overline{\mathbf{c}} \gamma_{5} \gamma_{i} \mathbf{u}\right)\left(\overline{\mathbf{u}} \gamma_{5} \mathbf{c}\right)\right]$ & $4238(31)(57)$ & $\mathrm{R}$ & $\mathrm{Y}(4260)$ \\
\hline $1^{--}$ & $\frac{1}{\sqrt{2}}\left[\left(\overline{\mathbf{s}} \gamma_{5} \gamma_{i} \mathbf{c}\right)\left(\overline{\mathbf{c}} \gamma_{5} \mathbf{s}\right)-\left(\overline{\mathbf{c}} \gamma_{5} \gamma_{i} \mathbf{s}\right)\left(\overline{\mathbf{s}} \gamma_{5} \mathbf{c}\right)\right]$ & $4405(31)(44)$ & $\mathrm{R}$ & \\
\hline $1^{--}$ & $\left(\overline{\mathbf{c}} \gamma_{i} \mathbf{c}\right)(\overline{\mathbf{c}} \mathbf{c})$ & $6411(25)(43)$ & $\mathrm{R}$ & \\
\hline $1^{--}$ & $\frac{1}{\sqrt{2}}\left\{\left[\mathbf{u}^{T} C \gamma_{5} \gamma_{i} \mathbf{c}\right]\left[\overline{\mathbf{u}} C \gamma_{5} \overline{\mathbf{c}}^{T}\right]+\left[\mathbf{u}^{T} C \gamma_{5} \mathbf{c}\right]\left[\overline{\mathbf{u}} C \gamma_{5} \gamma_{i}^{T} \overline{\mathbf{c}}^{T}\right]\right\}$ & $4267(68)(83)$ & $\mathrm{R}$ ? & $\mathrm{Y}(4260)$ \\
\hline $1^{--}$ & $\frac{1}{\sqrt{2}}\left\{\left[\mathbf{s}^{T} C \gamma_{5} \gamma_{i} \mathbf{c}\right]\left[\overline{\mathbf{s}} C \gamma_{5} \overline{\mathbf{c}}^{T}\right]+\left[\mathbf{s}^{T} C \gamma_{5} \mathbf{c}\right]\left[\overline{\mathbf{s}} C \gamma_{5} \gamma_{i}^{T} \overline{\mathbf{c}}^{T}\right]\right\}$ & $4449(40)(55)$ & $\mathrm{R}$ & \\
\hline $1^{++}$ & 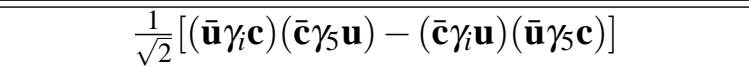 & $3895(27)(35)$ & $\mathrm{R}$ & $\mathrm{X}(3872)$ \\
\hline $1^{++}$ & $\frac{1}{\sqrt{2}}\left[\left(\overline{\mathbf{s}} \gamma_{i} \mathbf{c}\right)\left(\overline{\mathbf{c}} \gamma_{5} \mathbf{s}\right)-\left(\overline{\mathbf{c}} \gamma_{i} \mathbf{s}\right)\left(\overline{\mathbf{s}} \gamma_{5} \mathbf{c}\right)\right]$ & $4109(21)(32)$ & $\mathrm{R}$ & \\
\hline $1^{++}$ & $\frac{1}{\sqrt{2}}\left\{\left(\mathbf{u}^{T} C \gamma_{i} \mathbf{c}\right)\left(\overline{\mathbf{u}} C \gamma_{5} \overline{\mathbf{c}}^{T}\right)-\left(\mathbf{u}^{T} C \gamma_{5} \mathbf{c}\right)\left(\overline{\mathbf{u}} C \gamma_{i}^{T} \overline{\mathbf{c}}^{T}\right)\right\}$ & $3891(17)(21)$ & $\mathrm{R}$ & $\mathrm{X}(3872)$ \\
\hline $1^{++}$ & $\frac{1}{\sqrt{2}}\left\{\left(\mathbf{s}^{T} C \gamma_{i} \mathbf{c}\right)\left(\overline{\mathbf{s}} C \gamma_{5} \overline{\mathbf{c}}^{T}\right)-\left(\mathbf{s}^{T} C \gamma_{5} \mathbf{c}\right)\left(\overline{\mathbf{s}} C \gamma_{i}^{T} \overline{\mathbf{c}}^{T}\right)\right\}$ & $4134(19)(25)$ & $\mathrm{R}$ & \\
\hline $1^{+}$ & $\frac{1}{\sqrt{2}}\left[\left(\overline{\mathbf{u}} \gamma_{i} \mathbf{c}\right)\left(\overline{\mathbf{c}} \gamma_{5} \mathbf{s}\right)-\left(\overline{\mathbf{c}} \gamma_{i} \mathbf{S}\right)\left(\overline{\mathbf{u}} \gamma_{5} \mathbf{c}\right)\right]$ & $4007(34)(31)$ & $\mathrm{R}$ & \\
\hline $1^{+}$ & $\frac{1}{\sqrt{2}}\left\{\left(\mathbf{u}^{T} C \gamma_{i} \mathbf{c}\right)\left(\overline{\mathbf{s}} C \gamma_{5} \overline{\mathbf{c}}^{T}\right)-\left(\mathbf{u}^{T} C \gamma_{5} \mathbf{c}\right)\left(\overline{\mathbf{s}} C \gamma_{i}^{T} \overline{\mathbf{c}}^{T}\right)\right\}$ & $4015(25)(27)$ & $\mathrm{R}$ & \\
\hline
\end{tabular}

Table 1: Masses of the lowest-lying states of the molecular operators and the diquark-antidiquark operators. The column R/S denotes resonance $(\mathrm{R})$ or scattering $(\mathrm{S})$ states. The entries with empty slots in the last column are theoretical predictions [11, 12, 13].

\section{Concluding remarks}

Note that we are working in the quenched approximation which in principle is unphysical. However, our previous results on charmed baryon masses [19], charmed meson masses, and our predictions of $f_{D}$ and $f_{D_{s}}$ [20] are all in good agreement with the experimental values [21, 22, 23, 24]. This seems to suggest that it is plausible to use the quenched lattice QCD with exact chiral symmetry to investigate the mass spectra of the charmonium-like 4-quark meson operators, as a first step toward the unquenched calculations. The systematic error due to quenching can only be determined after we can repeat the same calculation with unquenched gauge configurations. (Note that Monte Carlo simulations of unquenched gauge configurations in lattice QCD with exact chiral symmetry, on the $20^{3} \times 40$ and $24^{3} \times 48$ lattices at $\beta=6.1$, still remains a challenge to the lattice community.) However, we suspect that the quenched approximation only results a few percent systematic error in the mass spectrum, especially for charmed mesons. Apparently, to gauge how well the quenched approximation in determining the mass spectrum of 4-quark meson operators is to see whether the predicted states in Table 1 (entries with empty slots in the last column) will be observed in high energy experiments. If it turns out that high energy experiments do not find any of these predicted states, then it might imply that: (i) the mass spectra of 4-quark mesons in quenched QCD could be dramatically different from those in unquenched QCD, or (ii) the constancy of the spectral weight for different volumes with the same lattice spacing (i.e., $W_{V_{1}} / W_{V_{2}} \simeq 1$ ) might not be sufficient to guarantee that it is a resonance, or both (i) and (ii). On the other hand, if the predicted states are confirmed by high energy experiments (even if our predicted masses turn out to be $5 \sim 8 \%$ off the experimental values), it would be a remarkable success for (quenched) lattice QCD with exact chiral symmetry. Obviously, no matter what is the outcome, it will be interesting. 


\section{Acknowledgement}

This work was supported in part by the National Science Council, Republic of China, under the Grant No. NSC94-2112-M002-016 (T.W.C.), and Grant No. NSC94-2119-M239-001 (T.H.H.), and by the National Center for High Performance Computation at Hsinchu, and the Computer Center at National Taiwan University.

\section{References}

[1] B. Aubert et al. [BABAR Collaboration], Phys. Rev. Lett. 90, 242001 (2003)

[2] S. K. Choi et al. [Belle Collaboration], Phys. Rev. Lett. 91, 262001 (2003)

[3] S. K. Choi et al. [Belle Collaboration], Phys. Rev. Lett. 94, 182002 (2005)

[4] B. Aubert et al. [BABAR Collaboration], Phys. Rev. Lett. 95, 142001 (2005)

[5] S. Uehara et al. [Belle Collaboration], Phys. Rev. Lett. 96, 082003 (2006)

[6] K. Abe et al., hep-ex/0507019.

[7] D. B. Kaplan, Phys. Lett. B 288, 342 (1992); Nucl. Phys. Proc. Suppl. 30, 597 (1993).

[8] R. Narayanan and H. Neuberger, Nucl. Phys. B 443, 305 (1995)

[9] H. Neuberger, Phys. Lett. B 417, 141 (1998)

[10] P. H. Ginsparg and K. G. Wilson, Phys. Rev. D 25, 2649 (1982)

[11] T. W. Chiu and T. H. Hsieh [TWQCD Collaboration], Phys. Rev. D 73, 094510 (2006)

[12] T. W. Chiu and T. H. Hsieh [TWQCD Collaboration], hep-ph/0603207.

[13] T. W. Chiu and T. H. Hsieh [TWQCD Collaboration], Phys. Rev. D 73, 111503 (2006)

[14] T. W. Chiu, Phys. Rev. Lett. 90, 071601 (2003); Nucl. Phys. Proc. Suppl. 129, 135 (2004).

[15] J. van den Eshof, A. Frommer, T. Lippert, K. Schilling and H. A. van der Vorst, Nucl. Phys. Proc. Suppl. 106, 1070 (2002)

[16] T. W. Chiu, T. H. Hsieh, C. H. Huang and T. R. Huang, Phys. Rev. D 66, 114502 (2002)

[17] H. Neuberger, Int. J. Mod. Phys. C 10, 1051 (1999)

[18] T. W. Chiu and T. H. Hsieh, Phys. Rev. E 68, 066704 (2003)

[19] T. W. Chiu and T. H. Hsieh, Nucl. Phys. A 755, 471 (2005)

[20] T. W. Chiu, T. H. Hsieh, J. Y. Lee, P. H. Liu and H. J. Chang, Phys. Lett. B 624, 31 (2005)

[21] W. M. Yao et al. [Particle Data Group], J. Phys. G 33, 1 (2006).

[22] M. Artuso et al. [CLEO Collaboration], Phys. Rev. Lett. 95, 251801 (2005)

[23] M. Artuso [CLEO Collaboration], hep-ex/0607074.

[24] S. Stone [CLEO Collaboration], hep-ex/0610026. 